Édition spéciale - L'agrobusiness en temps d'effondrement planétaire: approches critiques

Vol. 57, p. 270-275, jun. 2021. DOI: 10.5380/dma.v56i0.80870. e-ISSN 2176-9109

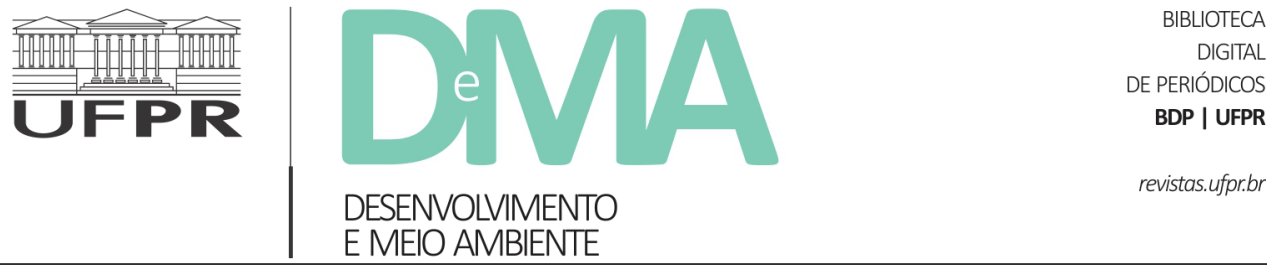

\title{
Quand des pesticides empoisonnent la recherche, la réglementation et la démocratie: L'Affaire Roundup à la lumière des Monsanto Papers
}

\section{Quando os agrotóxicos contaminam a pesquisa, a regulamentação e a democracia: o Caso Roundup à luz dos Monsanto Papers}

\section{When pesticides are poisoning research, regulation, and democracy: The Roundup Affair in Light of the Monsanto Papers}

Seralini' ${ }^{1}$, G.-E.; Douzelet, J. L'affaire Roundup à la lumière des Monsanto Papers.

Paris: Éditions Actes Sud, 2020.

Louise VANDELAC ${ }^{1,2^{*}}$

${ }^{1}$ Département de sociologie et Institut des sciences de l'environnement, Université du Québec à Montréal (UQAM), Canada.

${ }^{2}$ Collectif de recherche écosanté sur les pesticides, les politiques et les alternatives (CREPPA), Université du Québec à Montréal (UQAM), Canada.

*Contact: vandelac.louise@uqam.ca

Recension d'ouvrage reçu le 4 mai 2021, version finale acceptée le 1 juin 2021, publié dans 30 juin 2021.

Digne d'un roman de John Le Carré, où la réalité dépasserait de loin la fiction, «L'Affaire Roundup à la lumière des Monsanto Papers» dévoile de façon systématique les stratégies de Monsanto, désormais Bayer-Monsanto qui, connaissant les dangers du Roundup, premier pesticide au monde, a tout fait pour en masquer les effets délétères sur la

${ }^{1}$ Seralini, s'écrit bien depuis 2021 sans accent. santé et l'environnement. Cette firme a ainsi modelé le cadre même du débat et de l'évaluation publics tout en multipliant les coups tordus pour détruire la réputation des chercheurs ayant mis en évidence la toxicité de ces herbicides ainsi que l'étonnante mécanique de la firme pour le cacher. 
En moins de 200 pages, on comprend l'ampleur et la diversité des stratégies parfaitement orchestrées de création de discours pseudo-scientifiques, de collusion et de manipulation de l'information, passant notamment par des agences comme Science Media Center, destinées à abreuver des journalistes pressés, autant de manœuvres visant à influer sur les bases mêmes de la réglementation au détriment de la santé et de l'environnement. On est alors d'autant plus effaré par la gravité et le caractère vicieux des attaques visant à éroder la crédibilité et à empoisonner littéralement la vie de Seralini, ce chercheur de renom et universitaire reconnu, qui, auteur d'une douzaine d'essais et ancien expert scientifique à la Commission du génie biomoléculaire de 1998 à 2007, a été reçu en France Chevalier de l'Ordre National du Mérite pour l'ensemble de sa carrière en 2008 et s'est vu décerné, entre autres, aux États-Unis, le Prix Théo Colborn de l'Environmental Health Symposium, en 2016.

Si cet ouvrage identifie les sources des pressions politiques et des menaces, il expose surtout pour reprendre les mots des auteurs, «des techniques dignes d'un système mafieux que l'on ne pouvait soupçonner et dont nous avons maintenant les preuves: corruption active auprès d'éditeurs scientifiques, rédaction des conclusions sanitaires réglementaires en lieu et place des agences, fichages illégaux détaillés des personnalités susceptibles d'aider la firme, d'être influençable ou bien de la desservir» (Seralini \& Douzelet, 2020, p. 31) le tout avec preuves à l'appui.

C'est par un étonnant retour de boomerang d'une cour de justice États-Uniennes, qu'a pu être confirmé, à partir des documents mêmes de Monsanto, ce que Seralini et son équipe avaient découvert à savoir que «le Roundup contient des poisons cachés» (Seralini \& Douzelet, 2020, p. 28).

À l'avocate américaine venue le rencontrer à son laboratoire en Normandie pour discuter de «comment aborder le lien possible entre le Roundup, qui contient du glyphosate et ses nombreux clients atteints de cancers» pour avoir trop utilisé ce pesticide, il avait suggéré de "s'intéresser d'abord aux documents que Monsanto tient secrets sur les tests que la firme a déjà effectués sur le produit. Les suites de cette discussion», raconte-t-il, «auront des conséquences explosives à travers le monde» (p. 28).

Ces liens entre science, droit et intérêt public ont en effet permis d'obtenir plus de 2.5 millions de documents, échelonnés entre 1970 et 2018-2019, démontrant les agissements inqualifiables de cette firme pour cacher à tout prix la toxicité de cet herbicide afin de préserver ses marchés, au détriment des impacts sanitaires. Ainsi, plusieurs chapitres de cet ouvrage dont «Retractation by corruption» dévoilent comment Monsanto a payé un directeur de revue pour faire retirer l'article majeur de Seralini et de son équipe, article pourtant déjà accepté pour publication après révision par les pairs. L'objectif de la firme était de faire littéralement disparaître les résultats de cette étude indépendante pour éviter qu'elle soit relayée et que d'autres études scientifiques puissent s'en inspirer. Menée sur la vie entière de 200 rats avec 100.000 paramètres mesurés, cette expérience unique et ses images de rats aux énormes tumeurs déjà largement diffusés dans les médias démontrait pour la première fois, souligne Seralini, les effets du Roundup et des OGM sur la vie entière de mammifères vivants. Rappelons qu'alors, le Roundup n'avait jamais été testé à long terme et que les tests de Monsanto ayant permis la commercialisation des OGM ne duraient que trois mois sur seulement 80 des 400 rats testés dont 320 
servaient de contrôles ou témoins. D'où l'intérêt pour Seralini de poursuivre et d'amplifier cette expérience avec le même protocole et les mêmes rats, pour en montrer les effets à long terme. Résultats?

Des déficiences rénales et hépatiques mortelles emportaient les animaux au cours de leur deuxième année, avant même que les gosses tumeurs ne deviennent des cancers. En comparaison, des groupes de rats nourris sans pesticides, ni OGM, ne présentent pas de maladies chroniques et avaient de deux à cinq fois moins de tumeurs non cancéreuses et seulement beaucoup plus tard dans leur vie. (Seralini \& Douzelet, 2020, p.52)

Face à ces résultats saisissants, la firme ne se contente pas de retirer l'article, mais transforme l'étude Seralini en «évènement multimédia construit dans le but de répandre un maximum de publicité négative» (Seralini \& Douzelet, 2020, p. 114) révèlent avec force détails des chapitres entiers. Plus troublant encore, au moment où Seralini est invité à Londres pour faire une intervention au Parlement britannique, il est heurté dans le métro, par une valise sous le genou gauche, suivi aussitôt d'une désagréable piqûre. Quelques heures plus tard, il est victime, d'un «streptocoque multi-résistant aux antibiotiques, inconnu mais à l'effet foudroyant en moins de 24 heures» (Seralini \& Douzelet, 2020, p. 111), qui a failli le terrasser et lui coûter l'amputation d'une jambe, alors que de sévères séquelles exigeront de très nombreux mois de convalescence. Ce chapitre «Hasard ou parapluie Bulgare» nous apprend qu'au même moment, des membres du personnel de Monsanto célébraient et s'octroyaient des prix pour les vainqueurs des pires actions de dénigrement entreprises contre Seralini...
À partir des documents déclassifiés et notamment des 20.000 pages de ces Monsanto Papers où le nom de Seralini apparaît 55.952 fois, les deux auteurs nous livrent une analyse au scalpel des exactions de cette firme, éclairée par un témoignage lucide, douloureux et émouvant. C'est en effet à partir de «ce maelstrom insupportable» (Seralini \& Douzelet, 2020, p. 29) où il s'est retrouvé jusqu'à aujourd'hui, que Seralini \& Douzelet identifient les principaux ressorts de ces réseaux de compromissions et de complicités scientifiques, institutionnelles et médiatiques où, rares sont ceux et celles qui semblent comprendre l'ampleur des enjeux en cause et s'offusquer de voir le loup «gérer» ainsi la bergerie réglementaire.

L'histoire sociopolitique des sciences nous a appris à quel point, nombre de chercheurs dont les travaux pionniers ont mis en évidence les effets catastrophiques de certains produits sur la santé et l'environnement, menaçant dès lors la réputation et les profits des firmes impliquées, se sont vus littéralement traîner dans la boue par l'industrie, et souvent pendant des années, avec tous les dommages collatéraux imaginables, avant que l'importance de leurs contributions pour la santé publique et l'environnement ne soit pleinement reconnue. Ce fût le cas notamment de la biologiste Rachel Carson, auteure du célèbre ouvrage «Le printemps silencieux» (1962) sur les dommages des pesticides, et le cas aussi, parmi des centaines d'autres, du Dr Herbert Needleman dont les travaux marquants sur les ravages du plomb sur la santé les enfants, ont conduit à retirer le plomb de l'essence et des peintures. En 2015, même le réputé Centre de recherche international sur le cancer (CIRC), lié à l'Organisation mondiale de la santé (OMS), à la suite de son rapport sur le caractère génotoxique et cancérigène probable du glyphosate et des herbicides à base de glyphosate 
(HBG), a été l'objet d'une opération de torpillage de Bayer-Monsanto, au coût de 17 millions de dollars (Foucart \& Horel, 2017). Jamais toutefois n'a-t-on vu une multinationale s'acharner ainsi pendant plus de 15 ans contre un chercheur indépendant.

\section{Pourquoi un tel déchaînement?}

La réponse courte tient dans cette phrase choc de Donna Farmer, toxicologue en chef déjà chez Monsanto et toujours chargée des affaires réglementaires chez Bayer-Monsanto, prononcée au cours de son témoignage au tribunal de Californie dans le cadre des premiers procès de victimes de cancer non hodgkinien attribué au Roundup: «Nous ne pouvons pas dire que le Roundup ne cause pas de cancer. Nous n'avons pas fait les tests sur les produits formulés» (Seralini \& Douzelet, 2020, p. 88). Que signifient produits formulés? Ceux qui sont vendus et utilisés. Autrement dit, l'herbicide à base de glyphosate (HBG) contenant d'autres produits que le seul glyphosate. Comme le soulignent Seralini \& Douzelet (2020), cela était déjà vrai en 2003, elle le reconnaît dans les Monsanto Papers (Mongly 00922458) ${ }^{2}$, et elle l'avouait encore, en 2017, dans sa déposition judiciaire. Or, cet aveu crucial ajoutent-ils «sous-tend la stratégie de communication de la firme qui, encore une fois, tente de confondre glyphosate et Roundup - rappelons que le glyphosate est le seul testé à long terme par la firme au niveau réglementaire» (Seralini \& Douzelet, 2020, p. 88).

En termes clairs, le glyphosate déclaré aux agences réglementaires comme étant l'ingrédient actif de cet herbicide, laisse croire que les additifs, les éléments inertes et les surfactants, constituant environ $60 \%$ de la formulation commerciale utilisée, sont inoffensifs et ne méritent ni d'être rigoureusement évalués, ni d'être pris en compte dans les calculs de ventes et d'épandages, négligeant alors les effets de plus de la moitié des herbicides épandus. Comment expliquer que le glyphosate, jamais utilisé seul comme herbicide, soit le principal objet d'évaluations réglementaires? Ce sont les efforts concertés de la firme, désormais révélés, qui permettent de comprendre ce paradoxe. Seralini \& Douzelet (2020), soulignent que dès 2002, quand l'EPA a demandé des comptes aux gens de Monsanto, ils admirent alors que «Nous sommes à l'aise avec le glyphosate, mais vulnérables par rapport aux surfactants. Le glyphosate est OK, mais le produit formulé provoque des dégâts écrivait Heydens à Donna Farmer (Mongly 00885526)» (p. 102). Parmi ces surfactants, le POEA, pour lequel dès 2010, Monsanto développe un document de stratégie pour en cacher la toxicité (Mongly 02721133). «Donna Farmer réussit à faire autoriser ces toxiques comme inertes et donc confidentiels (Mongly 00878879)» [...] et précise que «On va rester sur nos positions de non-toxicité» $[\ldots]$ car «il est essentiel que toutes les intentions d'interdire ne se réfèrent pas au risque pour la santé humaine [...] sinon cela aurait un impact global sur la marque» (Mongly 0341522) (p. 90).

Mensonges et manipulations, alors même que des études démontrent que les HBG peuvent s'avérer jusqu'à 1.000 fois plus toxiques que l'ingrédient déclaré actif (Mesnage et al., 2014) et que les HBG, généralement composés d'environ $40 \%$ de glyphosate (Seralini \& Jungers, 2020), contiennent habi-

\footnotetext{
${ }^{2}$ Mongly pour Monsanto et glyphosate désigne le document numéroté des Monsanto Papers.
} 
tuellement de $10 \%$ à $20 \%$ de formulants chimiques, principalement des familles de molécules oxydées à base de pétrole, telles que les POEA, ainsi que des métaux lourds (arsenic, chrome, cobalt, plomb et nickel) (Defarge et al., 2018).

Les mots, on le voit, jouent ici un rôle primordial. Ainsi, qualifier les HBG de glyphosate, au point de confondre le produit utilisé avec l'élément déclaré actif par le fabricant, permet d'exempter de l'évaluation les autres composés toxiques des HBG. Cela conduit à d'absurdes polémiques où les instances réglementaires, prétendent toujours qu'il n'y a aucun problème avec le glyphosate, alors que 125.000 victimes américaines de cancer non hodgkinien l'attribuent bien au Roundup. Ce que confirme, aussi indirectement, l'entente hors-cours de 10.9 milliards de dollars U.S. avec Bayer-Monsanto. Toutefois, le second projet d'entente hors-cours de 2 milliards, proposé par Bayer pour éviter de nouvelles poursuites, a été rejeté le 26 mai 2021 par le juge Chhabria à la Cours de district de San Francisco. Le lendemain Bayer-Monsanto envisageait de cesser les ventes de Roundup pour usages résidentiels aux États-Unis.

Le caractère unique de cet ouvrage tient certes aux Monsanto Papers, éclairés par l'incomparable expérience scientifique de Gilles-Éric Seralini et au patient travail de décodage de Jérôme Douzelet, mais aussi aux prestations remarquables des avocats impliqués dans ce dossier et de plusieurs journalistes d'enquête. Notamment, Carey Gillam qui a joué un rôle clé dans toute l'affaire alors que des années plus tôt les trois ouvrages et documentaires de Marie-Monique Robin sur Monsanto ont «réussi à témoigner de l'innommable, transformant ainsi dans le monde entier une marque commerciale en marque de disgrâce» (Vandelac, 2018, p. 9).
L'importance de cet ouvrage tient aussi au marché colossal des HBG, premiers pesticides au monde, et aux OGM qui y sont liés, qui ont largement contribué à une concentration agroindustrielle aux allures de cartel, dominée par quelques firmes au pouvoir exorbitant: trois multinationales contrôlant $70 \%$ de l'agrochimie mondiale, quatre géants ayant la mainmise sur $60 \%$ des semences brevetées, quatre firmes régentant $90 \%$ du commerce mondial des céréales et trois compagnies détenant $50 \%$ de la machinerie agricole (ETC Group, 2019; Mooney, 2018).

L'intérêt suscité par le dossier global des pesticides tient aussi aux 385 millions de cas d'empoisonnement graves et non intentionnels aux pesticides par année dans le monde, parmi lesquels on compte 11.000 décès par an (Boedeker et al., 2020). Cela découle notamment de la hausse de $81 \%$ des ventes globales de pesticides, depuis 1990 , notamment en Asie et en Amérique du Sud (Boedeker et al., 2020), mais aussi des lacunes majeures de l'évaluation de ces pesticides, sur lesquelles cet ouvrage lève le voile. Commencent en effet à être révélées «les preuves presqu'uniques dans l'histoire du système de corruption de la science, de la règlementation et de l'information, des agences sanitaires, des gouvernements et des académies» soulignent les auteurs (Seralini \& Douzelet, 2020, p. 29), nous aidant ainsi à comprendre les enjeux et les ressorts d'un monde pesticides qui dépasse largement les agissements d'une seule firme. Cet ouvrage devrait avoir une résonnance toute particulière au Brésil, aux États-Unis, en Argentine et au Canada, ces grands producteurs d'Amérique de cultures OGM gavées d'HBG. Dès 1'automne 2021 des traductions anglaise, espagnole et portugaise de cet ouvrage seront disponibles. 


\section{Références}

Boedeker, W.; Watts, M.; Clausing, P. et al. The global distribution of acute unintentional pesticide poisoning: estimations based on a systematic review. BMC Public Health, 20,1875, 2020. doi: 10.1186/s12889-020-09939-0

Defarge, N.; Spiroux de Vendômois, J.; Seralini, G.E. Toxicity of Formulants and Heavy Metals in Glyphosate-Based Herbicides and Other Pesticides. Toxicology Reports, 5, 156-63, 2018. doi: 10.1016/j.toxrep.2017.12.025

ETC Group. Plate Tech-Tonics: Mapping Corporate Power in Big Food. Corporate concentration by sector and industry rankings by 2018 revenue. Val-David, Québec: ETC Group, 2019.

Foucart, S.; Horel, S. Monsanto papers: la guerre du géant des pesticides contre la science. Le Monde, 01 juin 2017. [En ligne]: <https://www.lemonde.fr/planete/article/2017/06/01/ monsanto-operation-intoxication_5136915_3244.html>.
Gillam, C. The Monsanto Papers. Deadly Secrets, Corporate Corruption, and One Man's Search for Justice. Island Press, 2021.

Mesnage, R.; Defarge, N.; Spiroux de Vendômois, J.; Seralini, G. E. Major pesticides are more toxic to human cells than their declared active principles. BioMed Research International, 179691, 2014. doi: 10.1155/2014/179691

Mooney, P. Blocking the Chain: Industrial Food Chain Concentration, Big Data Platforms and Food Sovereignty Solutions. Val-David et Berlin: ETC Group, 2018.

Seralini, G.E.; Jungers, G. Toxic Compounds in Herbicides without Glyphosate. Food and Chemical Toxicology, 146, 111770, 2020. doi: 10.1016/j.fct.2020.111770

Vandelac, L. Avaler des pesticides au risque d'être avalés par notre mutisme. Préface. In Robin, M.M. Le Roundup face à ses juges. Montréal: Éditions Écosociété, 2018, p. 9-25. 